\title{
Onkologische Betreuung
}

\section{Patienten attestieren ihren Urologen Bestnoten}

— Patienten mit Prostatakrebs fühlen sich von ihren Urologen bestens betreut. So lautet ein Fazit der diesjährigen Zwischenauswertung der bisher einzigen großen Versorgungsstudie zum Prostatakarzinom im deutschsprachigen Raum. Für den Präsidenten des Berufsverbands der Deutschen Urologen (BDU), Dr. Martin Bloch, ist das positive Votum der Patienten der sogenannten HAROW-Studie ein weiterer Beleg für die exzellente onkologische Kompetenz der niedergelassenen Urologen in Deutschland.

Nach dem nun veröffentlichten HAROW-Zwischenbericht 2010 schätzen die Patienten ihre behandelnden Urologen überaus positiv ein. Die Erhebung und Auswertung der Daten zur Arzt-PatientInteraktion erfolgte durch das Zentrum für Versorgungsforschung Köln (ZVFK) und attestiert den teilnehmenden Urologen eine hohe Einschätzung ihrer psychosozialen Versorgungsqualität und ärztlichen Empathie. Danach haben fast 90\% der Patienten einen festen Ansprechpartner in der urologischen Praxis. Die weitaus meisten fühlen sich unterstützt, wertgeschätzt und verständlich informiert. Über $40 \%$ der Urologen sind sogar außerhalb der
Sprechzeiten für ihre Patienten da. Über $80 \%$ der Befragten geben an, genügend Zeit für wichtige therapeutische Entscheidungen zu bekommen. Knapp $96 \%$ der Patienten werden von ihren niedergelassenen Urologen über die verschiedenen Therapieoptionen informiert und $80 \%$ fühlen sich in die Therapieentscheidung einbezogen.

„Die Patienten der Studie haben eine hervorragende Meinung von ihren behandelnden Urologen. In Schulnoten ausgedrückt ist das für die meisten Arzt-Patienten-Interaktionen ein ,sehr gut' und zeigt, dass niedergelassene Urologen auch in der Wahrnehmung der Patienten exzellente Onkologen sind“, sagt Dr. Bloch. Der wissenschaftliche Leiter der Studie, Prof. Lothar Weißbach, betont: „Die Studienleitung hat sich sehr über dieses Ergebnis gefreut. Jetzt wird sie mit den Kolleginnen und Kollegen des ZVFK und des wissenschaftlichen Studienbeirats überlegen, wie Defizite bei der Zweitmeinung, der Einbeziehung der Selbsthilfegruppen und der Aushändigung guter Informationsmaterialien zu beheben sind." 